\title{
cris Umbilical Cord Blood Acid-Base Status in
Newborns with Congenital Heart Disease in a
Cardiovascular Referral Center in Colombia
}

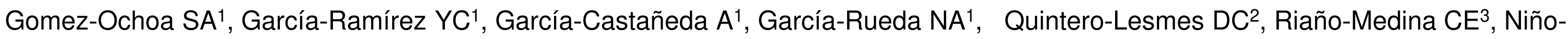
Tovar $\mathrm{A}^{1,4}$.

1. Medicine School, Health Faculty, Universidad Industrial de Santander, Bucaramanga, Colombia. 2. Epidemiology and Research Unit. Fundación Cardiovascular de Colombia, Bucaramanga, Santander, Colombia. 3. Gynecology and Obstetrics Department, Fundación Cardiovascular de Colombia, Bucaramanga, Santander, Colombia. 4. Neonatal Intensive Care Unit, Fundación Cardiovascular de Colombia, Bucaramanga, Santander, Colombia.

Background and aims: Congenital Heart Diseases (CHD) are defined as defects of the cardiac architecture that interfere with blood flow dynamic, with a variable effect in fetal circulation and a significant morbidity, however, their pathophysiology and hemodynamic impact remain not fully understood. This study represents the first to evaluate the impact of CHD in umbilical cord blood acid-base status ${ }^{(1,2)}$.

Methods: Analytic retrospective study, performed in the Fundación Cardiovascular de Colombia. Newborns with a post-natal echocardiographic diagnosis of CHD born during the period 2007-2015 were included. The CHD was characterized using a pathophysiological classification. Demographic, clinical, imaging and laboratory variables, including the umbilical cord blood acid-base status, were retrieved from the clinical records.

Table 1. Pathophysiological classification of Congenital Heart Diseases

\begin{tabular}{|c|c|c|}
\hline Category & Acronym & CHD \\
\hline $\begin{array}{l}\text { CHD with increased pulmonary } \\
\text { blood flow }\end{array}$ & IPBF & $\begin{array}{c}\text {-Partial anomalous pulmonary venous drainage } \\
\text {-Atrial septum defects (ASD) } \\
\text {-AV septum defects } \\
\text {-Ventricular septum defects (VSD) } \\
\text {-Persistent truncus arteriosus } \\
\text {-Patent ductus arteriosus }\end{array}$ \\
\hline $\begin{array}{l}\text { CHD with decreased } \\
\text { pulmonary flow }\end{array}$ & DPBF & $\begin{array}{c}\text { - Pulmonary valve stenosis with ASD } \\
\text { - Pulmonary stenosis with VSD (Tetralogy of } \\
\text { Fallot) } \\
\text {-Tricuspid atresia } \\
\text {-Ebstein anomaly of the tricuspid valve } \\
\text {-Single ventricle with pulmonary stenosis }\end{array}$ \\
\hline $\begin{array}{c}\text { CHD with obstruction to blood } \\
\text { progression and no } \\
\text { septal defects }\end{array}$ & OBNSD & $\begin{array}{l}\text {-Pulmonary stenosis } \\
\text {-Aortic stenosis } \\
\text {-Coarctation of the aorta }\end{array}$ \\
\hline $\begin{array}{l}\text { CHD so severe as to be } \\
\text { incompatible with postnatal } \\
\text { blood circulation }\end{array}$ & NCBC & $\begin{array}{l}\text {-Pulmonary atresia } \\
\text {-Aortic/Mitral atresia } \\
\text {-Complete transposition of the great arteries } \\
\text {-Total anomalous pulmonary venous drainage }\end{array}$ \\
\hline CHD silent until adult age. & SUAA & $\begin{array}{c}\text { - Bicuspid aortic valve } \\
\text {-Congenital anomalies of coronary arteries } \\
\text {-Wolff-Parkinson-White Syndrome } \\
\text {-Congenitally corrected transposition of the great } \\
\text { arteries }\end{array}$ \\
\hline
\end{tabular}

Results: 82 patients were included, with a total of 136 heart defects. The CHD classification and the description of the population are summarized in the tables. The largest group was the IPBF $(n=58 ; 42,6 \%)$, while only 1 case was reported in the SUAA one.
Moreover, patients in the NCBC group were significantly older than the ones in DPBF $(p=0.03)$ and OBNSD $(p=0.04)$. Median $\mathrm{pH}$ value was 7.3 (IQR 7.2-7.35), being similar among the groups, as the rest of cord blood variables. Death rate was homogeneous among the groups, having DPBF the highest one $(48,2 \%)$.

Table 2. Demographic Variables and Umbilical Cord Blood Acid-base Status by CHD Group

\begin{tabular}{|c|c|c|c|c|c|}
\hline Variable & $\begin{array}{c}\text { IPBF } \\
n=58\end{array}$ & $\begin{array}{l}\text { DPBF } \\
n=27\end{array}$ & $\begin{array}{l}\text { OBNSD } \\
n=37\end{array}$ & $\begin{array}{l}\text { NCBC } \\
n=13\end{array}$ & $\begin{array}{l}\text { SUAA } \\
n=1\end{array}$ \\
\hline $\begin{array}{l}\text { Gestational Age } \\
\text { (Weeks) }\end{array}$ & 38 (IQR 37 - 38.5) & $\begin{array}{c}38(\text { IQR } \\
36.2-38.1)\end{array}$ & $\begin{array}{c}38 \text { (IQR } 37 \text { - } \\
38.5 \text { ) }\end{array}$ & $\begin{array}{c}38.75 \text { (IQR } \\
38-39)\end{array}$ & 37 (IQR 37 - 37) \\
\hline Males & $25(43.86 \%)$ & $13(52 \%)$ & 19 (52.78\%) & $8(57.1 \%)$ & $0(0 \%)$ \\
\hline Weight (Grams) & $\begin{array}{c}2930 \text { (IQR } 2700 \text { - } \\
3260 \text { ) }\end{array}$ & $\begin{array}{c}3000 \text { (IQR } \\
2650- \\
3240)\end{array}$ & $\begin{array}{c}2960 \text { (IQR } \\
2700-3150)\end{array}$ & $\begin{array}{c}3065 \text { (IQR } \\
2700- \\
3300)\end{array}$ & $\begin{array}{c}2960 \text { (IQR } 2700 \\
-3240)\end{array}$ \\
\hline$p H$ & $\begin{array}{c}7.29 \text { (IQR 7.2 - } \\
7.36 \text { ) }\end{array}$ & $\begin{array}{c}7.3 \text { (IQR } 7.2 \\
-7.36)\end{array}$ & $\begin{array}{c}7.3 \text { (IQR } 7.23 \\
-7.375)\end{array}$ & $\begin{array}{l}7.28(\mathrm{IQR} \\
7.2-7.35)\end{array}$ & -- \\
\hline PCO2 $(\mathrm{mm} \mathrm{Hg})$ & $\begin{array}{c}42.5 \text { (IQR } 35.1 \text { - } \\
53.2 \text { ) }\end{array}$ & $\begin{array}{l}42.2 \text { (IQR } \\
33.3-47)\end{array}$ & $\begin{array}{c}41.4 \text { (IQR } \\
34.25- \\
52.85)\end{array}$ & $\begin{array}{l}50.9 \text { (IQR } \\
39.3-56)\end{array}$ & -- \\
\hline PO2 $(\mathrm{mm} \mathrm{Hg})$ & 33 (IQR 23 - 46) & $\begin{array}{c}31 \text { (IQR } 28- \\
37.1)\end{array}$ & $\begin{array}{c}32.5 \text { (IQR } 22 \\
-47)\end{array}$ & $\begin{array}{c}26.7 \text { ( IQR } \\
16-39)\end{array}$ & -- \\
\hline $\begin{array}{l}\text { Base excess } \\
(\mathrm{mEq} / \mathrm{L})\end{array}$ & $\begin{array}{c}(-) 3.6[\text { [IQR (- } \\
) 7.05-(-) 2.35]\end{array}$ & $\begin{array}{c}(-) 3.6[\text { IQR } \\
(-) 7.6-(-) \\
2.9]\end{array}$ & $\begin{array}{c}(-) 4.35[\mathrm{IQR} \\
(-) 6.6-(-) \\
2.37]\end{array}$ & $\begin{array}{c}23.3(\mathrm{IQR} \\
21.17- \\
24.82)]\end{array}$ & -- \\
\hline HCO3 (mEq/L) & $\begin{array}{c}22.65 \text { (IQR } 19.5 \text { - } \\
24.5 \text { ) }\end{array}$ & $\begin{array}{c}22.5(\mathrm{IQR} \\
18.6-23.2)\end{array}$ & $\begin{array}{c}22.9(\text { IQR } \\
20.5-24.5)\end{array}$ & $\begin{array}{c}23.3(\text { IQR } \\
21.3-24.7)\end{array}$ & -- \\
\hline $\begin{array}{l}\text { Lactate } \\
(\mathrm{mmol} / \mathrm{L})\end{array}$ & $\begin{array}{c}2.55 \text { (IQR } 2.1 \text { - } \\
4.1 \text { ) }\end{array}$ & $\begin{array}{c}2.65 \text { (IQR } 2 \\
-3.6)\end{array}$ & $\begin{array}{c}2.35 \text { (IQR } 1.7 \\
-4)\end{array}$ & $\begin{array}{c}2.5 \text { (IQR } 2.3 \\
-3.8)\end{array}$ & -- \\
\hline
\end{tabular}

Conclusions: There were not significant differences among the groups regarding the acid-base status, this could be attributed to the potential compatibility of fetal circulation and some cardiac structural abnormalities, however, our small sample may have influenced this result, highlighting the need of larger studies.

\section{References:}

1.) Zhan $Q$, Wang $X, Y u$ J, Fan $Y$. Umbilical cord blood acid-base status in pregnancy with congenital heart disease. Acta Anaesthesiol Scand. 2014;58(7):851-7.

2.) Thiene G, Frescura C. Anatomical and pathophysiological classification of congenital heart disease. Cardiovasc Pathol. 2010;19(5):259-74. 